\title{
Association between Psychiatric Disorders and Glomerular Disease
}

\author{
Hailey E. Desmond ${ }^{\mathrm{a}} \quad$ Clare Lindner $^{\mathrm{a}} \quad$ Jonathan P. Troost ${ }^{\mathrm{b}}$ Zack Held $^{\mathrm{c}}$ \\ Andrea Callaway ${ }^{d}$ Gia J. Oh ${ }^{e}$ Richard Lafayette ${ }^{f}$ Michelle O'Shaughnessy $^{g}$ \\ Matthew Elliott ${ }^{\mathrm{h}}$ Sharon G. Adler ${ }^{\mathrm{i}}$ Elaine S. Kamil ${ }^{j} \quad$ Anne Pesenson $^{\mathrm{k}}$ \\ David T. Selewskil Patrick E. Gipson ${ }^{a}$ m Noelle E. Carlozzin ${ }^{n}$ Debbie S. Gipson ${ }^{a}$ \\ Susan F. Massengillc

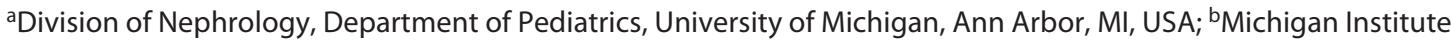 \\ for Clinical and Health Research, University of Michigan, Ann Arbor, MI, USA; 'Division of Pediatric Nephrology, \\ Department of Pediatrics, Levine Children's Hospital, Atrium Health, Charlotte, NC, USA; dephrotic Syndrome \\ Foundation, Alamo, CA, USA; 'Department of Pediatric Nephrology, Randall Children's Hospital at Legacy Emanuel \\ Medical Center, Legacy Health, Portland, OR, USA; 'Division of Nephrology, Stanford University School of Medicine, \\ Palo Alto, CA, USA; 9 University College Cork, Cork University Hospital, Cork, Ireland; hMetrolina Nephrology \\ Associates, Charlotte, NC, USA; 'Division of Nephrology and Hypertension, Los Angeles Biomedical Research \\ Institute at Harbor-University of California, Torrance, CA, USA; jDivision of Pediatric Nephrology, Department of \\ Pediatrics, Cedars-Sinai Medical Center, David Geffen School of Medicine at the University of California, Los Angeles, \\ CA, USA; 'The Polyclinic, Seattle, WA, USA; 'Division of Nephrology, Department of Pediatrics, Medical University \\ of South Carolina, Charleston, SC, USA; 'mivision of Nephrology, Department of Internal Medicine, University of \\ Michigan, Ann Arbor, MI, USA; "Department of Physical Medicine and Rehabilitation, University of Michigan, Ann \\ Arbor, MI, USA
}

\section{Keywords}

Psychiatric disorder - Anxiety · Glomerular disease .

Nephrotic syndrome $\cdot$ End-stage kidney disease $\cdot$ Electronic health record

\begin{abstract}
Introduction: Patients with chronic health conditions, particularly chronic kidney disease, are at heightened risk for psychiatric disorders; yet, there are limited data on those with primary glomerular disease. Methods: This study included patients with glomerular disease enrolled in the kidney research network multisite patient registry. Registry data include encounter, diagnoses, medication, laboratory, and vital signs data extracted from participants' electronic
\end{abstract}

health records. ICD-9/10 diagnosis codes were used to identify a subset of psychiatric disorders focused on anxiety, mood, and behavioral disorders. Time-varying Cox proportional hazard models were used to analyze time from the onset of kidney disease to diagnosis of psychiatric disorder. Adjusted models retained significant covariates from the full list of potential confounders, including age, sex, race, ethnicity, time-varying treatment, the estimated glomerular filtration rate, and proteinuria (urine protein-to-creatinine ratio [UPCR]). Analogous models examined diagnosis of psychiatric disorder as a predictor of time to end-stage kidney disease (ESKD). Results: Data were available for 950 participants, with a median of 58 months of follow-up. 110 (12\%) participants were diagnosed with psychiatric disorder during the follow-up. The estimated rate of psychiatric diagno- karger@karger.com www.karger.com/gdz

Karger $\stackrel{\text { ' }}{5}$

BOPEN ACCESS
(C) 2021 The Author(s)

Published by S. Karger AG, Basel

This is an Open Access article licensed under the Creative Common Attribution-NonCommercial-4.0 International License (CC BY-NC) (http://www.karger.com/Services/OpenAccessLicense), applicable to the online version of the article only. Usage and distribution for commercial purposes requires written permission.
Correspondence to:

Hailey E. Desmond, heturner@med.umich.edu 
sis after kidney disease was 14.7 cases per 1,000 person-years and was highest among those of adolescent age at the time of kidney disease diagnosis. Adjusted analyses found adolescent age (vs. adult, hazard ratio $[H R]=3.11,95 \%$ confidence interval $[\mathrm{Cl}]$ 1.87-5.17) and Asian race (vs. white, $\mathrm{HR}=0.34$, $95 \% \mathrm{Cl} 0.16-0.71$ ) were associated with psychiatric diagnosis. A higher UPCR per 1 log unit (HR 1.13, 95\% Cl 1.01-1.27) and a higher total number of oral medications were associated with psychiatric disorder $(p<0.001)$. Psychiatric diagnosis was also associated with progression to ESKD (HR = $2.45,95 \% \mathrm{Cl} 1.53-3.92$ ) in adjusted models. Discussion/Conclusion: Psychiatric disorders were documented in approximately one-eighth of patients with glomerular disease and correlated with clinical disease characteristics such as age, race, proteinuria, and oral medication burden. These findings suggest mental health screening is warranted in patients of all ages with glomerular disease.

(c) 2021 The Author(s)

Published by S. Karger AG, Basel

\section{Introduction}

Improved outcomes in the treatment and management of chronic illness have led to an increase in the number of children and adults living with chronic medical conditions. Previous research into chronic diseases, such as diabetes, chronic obstructive pulmonary disease, asthma, and sickle cell disease, has shown that ongoing treatment demands and disease progression can contribute to emotional and behavioral problems. Studies have demonstrated that patients with chronic illnesses experience higher levels of depressive symptoms than their healthy peers [1-5]. In addition, psychological distress in a chronically ill population has been consistently associated with poor health outcomes and an increase in somatic complaints across various diseases, including diabetes, chronic obstructive pulmonary disease, and sickle cell disease $[4,6-10]$.

Disease-specific differences have been reported across the age spectrum. For instance, youth with poorly controlled asthma have significantly higher odds than those with mild or well-controlled asthma of experiencing affective and anxiety problems as well as more frequent conduct disorders $[11,12]$. Likewise, research has shown that when compared to healthy peers, children and adolescents with asthma show a higher incidence of internalizing and externalizing behaviors [6, 7], while adults consistently express higher levels of anxiety (specifically panic disorder) and depressive disorders [13-16]. In cystic fibrosis, depression has been documented in similar

Association between Psychiatric

Disorders and Glomerular Disease ranges in children (8-29\%) and adolescents and adults (13-33\%). Similarly, the proportion of children and adults with cystic fibrosis and anxiety ranges between 30 and $33 \%$ [17-19]. As a comparison, healthy cohort data support an expected frequency of depression in 3\% of children and adolescents and $7 \%$ of adults and of anxiety in $7 \%$ of children and $19 \%$ of adults [20,21].

The impact of disease-specific psychological stressors has been a focus in the diabetes literature across the life span. High levels of diabetes-related stress had a significant impact on metabolic control and adherence and led to worse outcomes for patients [22-24]. Additionally, diabetes has consistently been linked to a $25 \%$ increase in the rate of depression across the life span $[25,26]$.

While there is a body of evidence demonstrating the negative effect of end-stage kidney disease (ESKD) on overall mental health [27-29], studies focusing on patients with pre-ESKD chronic kidney disease (CKD) are more limited and conflicting. In community clinical settings and the Veteran Affairs Health System (VAHS) settings, studies have found that between 21 and $30 \%$ of individuals with pre-ESRD and CKD have depression [30, 31]. However, a prior study using the National Health and Nutrition Examination Survey (NHANES) 2005-2006 found only $7 \%$ of participants with CKD to have depression. The Chronic Kidney Disease in Children (CKiD) cohort found approximately $7 \%$ of participating children either had elevated depressive symptoms or were being treated for depression, and further concluded that depression correlated with lower health-related quality of life [32].

Clearly, patients with chronic conditions are at a heightened risk for psychiatric disorders and there are disease-specific differences that may impact psychological distress and treatment outcomes. Like many chronic diseases, we hypothesized that glomerular disease has both a physical and psychological impact on patients. Possible causes of this include medication burden, side effects of medications, burden of dietary restrictions, and chronic illness fatigue. In addition, the unpredictable and uncertain nature of glomerular disease is a further likely factor. Therefore, the goal of this study was to utilize the Kidney Research Network (KRN) patient registry to explore the occurrence of psychiatric disorders in patients with glomerular disease, the potential associations between psychiatric diagnosis and demographic and clinical characteristics, and the relationship between psychiatric diagnosis and progression to ESKD. 
Fig. 1. Flow diagram of included partici-

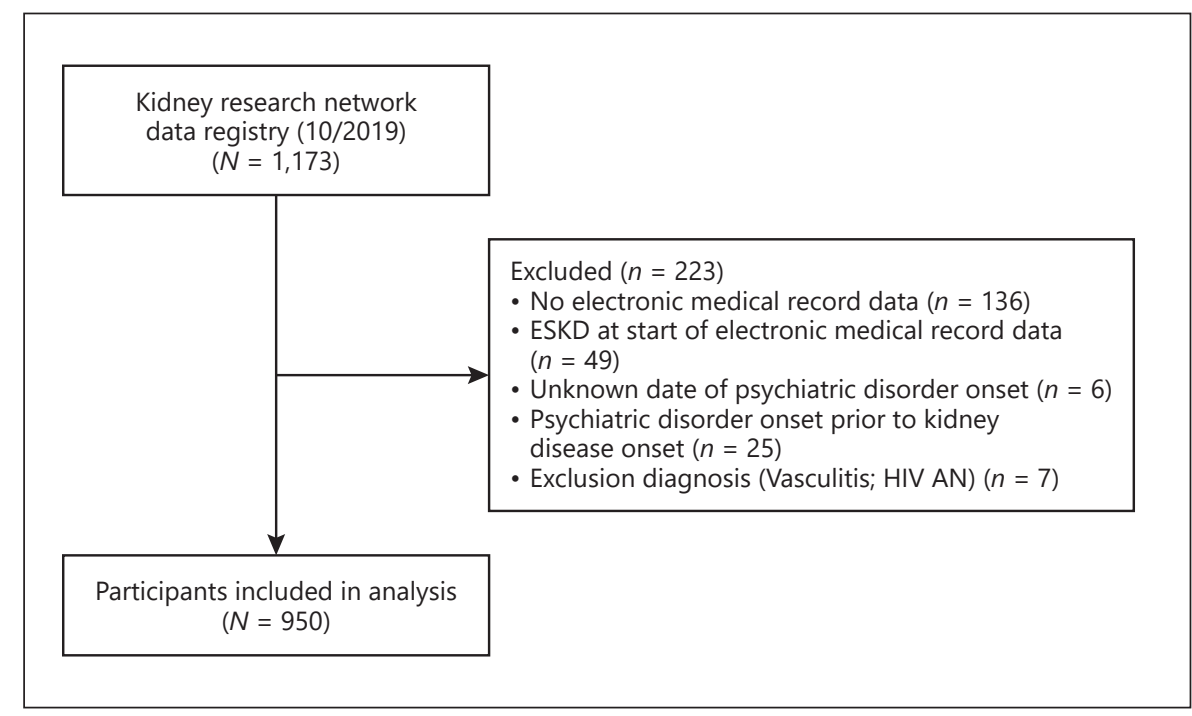
pants.

\section{Materials and Methods}

\section{Participants and Data Source}

The KRN is an ongoing registry of patients with proteinuric kidney disease (glomerular disease) from 7 clinical centers in the USA that began enrollment in August 2015 [33]. Patients enrolled in the KRN consented to share retrospective (prior to enrollment) and prospective (monthly post-enrollment) data directly reported from study personnel as well as extracted from their electronic health records (EHRs). The data collection has been previously described and includes demographics, diagnoses including provider confirmed primary kidney diagnosis, kidney biopsy reports, laboratory values, vital signs, medications, and hospitalizations [33]. Patients could consent to join the registry at any point in their kidney disease course, but retrospective EHR data were available from the time the patient entered the participating nephrology site EHR environment. The study protocol was reviewed and approved by the University of Michigan Institutional Review Board which serves as IRB of record for all enrolling sites. All patient participants or legal guardians provided informed consent prior to entry into the study registry.

For this analysis, we excluded participants with ESKD prior to their first EHR data capture event in the KRN data registry. Also excluded were those with ANCA vasculitis or HIV-associated nephropathy due to the possibility of CNS manifestations of underlying disease as an unpredictable variable in this population. Additionally, while 6 of the $7 \mathrm{KRN}$ sites provide EHR data extracts, as of this study analysis, one site provided kidney disease-focused data via manual data entry alone. Participants $(n=136)$ from this site were excluded due to the unlikely capture of the primary outcome of interest. Participants with diagnosis of psychiatric disorder prior to the onset of kidney disease were excluded from these analyses.

\section{Outcomes}

The primary outcome of interest was psychiatric disorder diagnosis. Psychiatric diagnoses were limited to anxiety (adjustment disorder, generalized anxiety disorder, obsessive compulsive dis- order, panic disorder, post-traumatic stress disorder, and other/ unspecified anxiety disorder), mood disorders (bipolar disorder, depressive disorders, and other/unspecified mood disorder), and behavioral disorders (attention deficit hyperactivity disorder, conduct disorder, intermittent explosive disorder, oppositional defiant disorder, and other/unspecified behavioral disorder). Diagnosis was defined as the date of the first occurrence of psychiatric disorder ICD-9/10 code (details in online suppl. Table 1; see www. karger.com/doi/10.1159/000516359 for all online suppl. material).

Secondary analyses examined diagnosis of psychiatric disorder during the period of observation as a potential predictor of progression to ESKD, defined as initiation of chronic dialysis $(\geq 60$ days), kidney transplant, chronically low estimated glomerular filtration rate (eGFR) (eGFR $<15$ for $\geq 60$ days), or ICD-9/10 codes of stage $5 \mathrm{CKD}$. An eGFR was calculated using the creatininebased modified CKiD formula in children and the CKD-EPI equation in adults $[34,35]$.

\section{Statistical Analyses}

The cohort was described using frequencies and percentages for categorical variables and medians with interquartile ranges for continuous variables. Stratified summary descriptions were provided by occurrence of psychiatric diagnosis after the onset of kidney disease. $p$ values comparisons were made with $\chi^{2}$ tests for categorical and Kruskal-Wallis tests for continuous characteristics. Kaplan-Meier plots were used to display the time from the onset of kidney disease to diagnosis of psychiatric disorder by age, and subset analyses were repeated for (1) anxiety disorder, (2) mood disorder, and (3) behavioral disorder. Incidence of diagnosis was quantified using a person-time denominator.

Time-varying Cox proportional hazard models were used to test for predictors of psychiatric diagnosis. Predictors of interest included age, sex, race, ethnicity, kidney diagnosis (focal segmental glomerulosclerosis [FSGS] vs. minimal change disease [MCD] vs. membranous nephropathy vs. nephrotic syndrome - not biopsied vs. IgA nephropathy vs. other), eGFR, urine protein-to-creatinine ratio (UPCR), immunosuppressive therapy (IST), insurance type, and the age-group. Participants with C1q nephropathy $(n=$ 
Table 1. Descriptive statistics of participants by diagnosis of psychiatric disorder in the KRN registry analysis sample $(n=943)$

\begin{tabular}{|c|c|c|c|c|}
\hline Age at kidney disease onset, years, median (IQR) & $18(5,40)$ & $15(5,35)$ & $19(4,41)$ & 0.004 \\
\hline Children ( $0-12$ years), $n(\%)$ & $395(42)$ & $46(42)$ & $349(42)$ & \\
\hline Adolescents (13-17 years), $n(\%)$ & $76(8)$ & $18(16)$ & $58(7)$ & \\
\hline Adults $(18+$ years $), n(\%)$ & $479(50)$ & $46(42)$ & $433(52)$ & \\
\hline White & $524(55)$ & $76(69)$ & $448(53)$ & \multirow[t]{5}{*}{0.01} \\
\hline Black & $104(11)$ & $10(9)$ & $94(11)$ & \\
\hline Asian & $156(16)$ & $8(7)$ & $148(18)$ & \\
\hline Other & $139(15)$ & $15(14)$ & $124(15)$ & \\
\hline Unknown/missing & $27(3)$ & $1(1)$ & $26(3)$ & \\
\hline Hispanic ethnicity, $n(\%)$ & $183(19)$ & $16(15)$ & $167(20)$ & 0.12 \\
\hline NS-not biopsied* & $143(15)$ & $9(8)$ & $134(16)$ & \multirow{3}{*}{0.003} \\
\hline IgA nephropathy & $156(16)$ & $11(10)$ & $145(17)$ & \\
\hline Other** & $133(14)$ & $17(15)$ & $116(14)$ & \\
\hline $\begin{array}{l}\text { eGFR closest to kidney disease onset, } \mathrm{mL} / \mathrm{min} / 1.73 \mathrm{~m}^{2} \text {, } \\
\text { median (IQR) }\end{array}$ & $87(43,121)$ & $102(64,133)$ & $83(39,120)$ & $<0.001$ \\
\hline UPCR closest to kidney disease onset, g/g, median (IQR) & $2.1(0.6,6.2)$ & $2.8(0.5,6.8)$ & $2.1(0.6,6.2)$ & 0.38 \\
\hline Ever treated with steroids, $n(\%)$ & $743(78)$ & $86(78)$ & $657(78)$ & 0.66 \\
\hline Ever treated with other ISTs, $n(\%)$ & $641(67)$ & $66(60)$ & $575(68)$ & 0.08 \\
\hline Observation duration, months median (IQR) & $58(27,99)$ & $86(51,122)$ & $57(23,93)$ & $<0.001$ \\
\hline
\end{tabular}

$p$ values from $\chi^{2}$ tests for categorical and Kruskal-Wallis tests for continuous characteristics. eGFR, estimated glomerular filtration rate; FSGS, focal segmental glomerulosclerosis; IQR, interquartile range; IST, immunosuppressive therapy; NS, nephrotic syndrome; UPCR, urine protein-to-creatinine ratio; KRN, kidney research network. ${ }^{*}$ Renal biopsy is not always first line care for childhood NS. ${ }^{*}$ Other diagnoses include Alport syndrome (49), SLE class V (29), C3 glomerulopathy (25), amyloidosis (5), congenital NS (5), immune complex-mediated glomerulonephritis (4), SLE class IV (3), fibrillary glomerulonephritis (2), interstitial nephritis (2), SLE class III (2), crescentic glomerulonephritis (1), glomerulonephritis with mesangial deposits and crescents (1), HSP nephritis (1), light chain nephropathy (1), MPGN with cryoglobulinemic glomerulonephritis (1), SLE class II (1), SLE class VI (1).

11) were reclassified as FSGS; participants with mesangial proliferative glomerulonephritis $(n=6)$ or IgM nephropathy $(n=17)$ were reclassified as MCD. Analyses were censored at the onset of ESKD. Hazard ratios (HRs) and 95\% confidence intervals (CIs) are reported. All variables were initially tested as unadjusted, univariable predictors of psychiatric diagnosis. Any variable significant with $p<0.20$ was included in a backward multivariable model selection. Nonsignificant variables were removed in reverse order of $p$ value until all remaining variables in the model were significant at $p<0.05$. Given the expected correlation between age, eGFR, UPCR, and diagnosis, multicollinearity of these variables was assessed using variance inflation factors (VIFs) from a single multivariable model of the 4 covariates. Any variable with $\mathrm{VIF}>2.5$ was excluded from model building; if all indicators showed $\mathrm{VIF}<2.5$, then all were included in model building. Analogous time-varying Cox proportional models were fit modeling time from the onset of kidney disease to ESKD. In these models, diagnosis of psychiatric disorder was included as a covariate to test for an association between psychiatric disorders and progression to ESKD. Analyses

Association between Psychiatric

Disorders and Glomerular Disease were completed in SAS ${ }^{\circledR}$ (version 9.4; SAS Institute Inc, Cary, NC, USA).

A sensitivity analysis was conducted for the primary outcome of psychiatric disorder excluding the cases with lupus nephritis. No significant differences in estimates were identified (online suppl. Table 3).

\section{Results}

\section{Sample}

As of October 2019, there were 1,173 participants enrolled in the KRN registry. After excluding subjects not fully enrolled or meeting other exclusion criteria, 950 participants contributed to this analysis sample as shown in Figure 1. 
Fig. 2. Time to diagnosis of any psychiatric disorder $(n=950$ participants; $n=110$ events; rate $=14.7$ cases per 1,000 personyears).
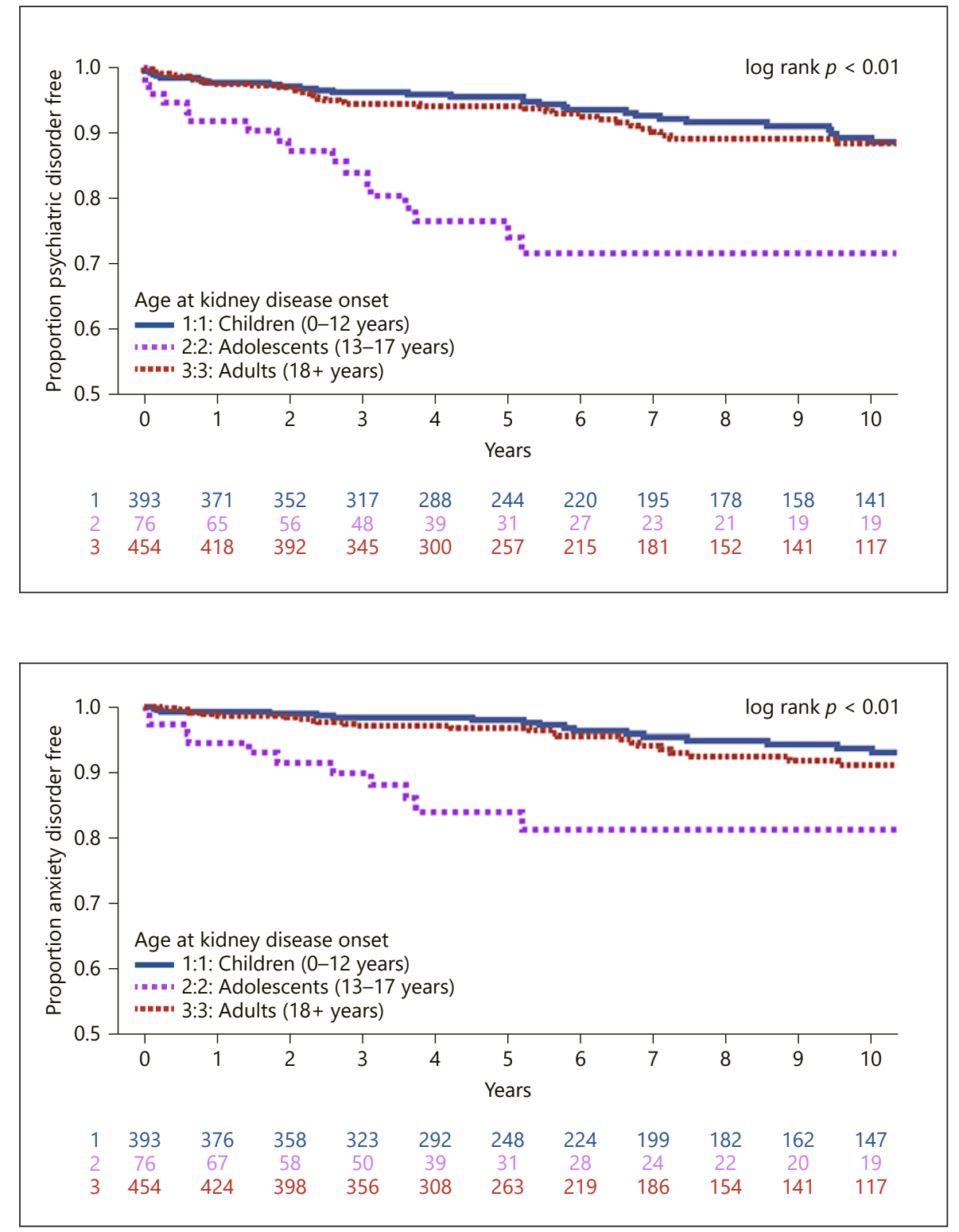

Fig. 3. Time to diagnosis of anxiety disorder $(n=950$ participants; $n=75$ events; rate $=9.8$ cases per 1,000 person-years) .

\section{Psychiatric Disorders}

The estimated rate of psychiatric diagnosis was 14.7 cases per 1,000 person-years. Time to diagnosis of any psychiatric disorder is shown as a Kaplan-Meier plot in Figure 2. Adolescent participants were more likely to be diagnosed with a psychiatric disorder (33.4 cases per 1,000 person-years) than children (13.3 cases per 1,000 person-years) or adults (13.2 cases per 1,000 personyears) $(\log$-rank $p<0.01)$.

Analyses were repeated for time to diagnosis in each disorder subcategory: anxiety shown in Figure 3, mood shown in Figure 4, and behavioral disorders shown in online suppl. Figure 1. Adolescents had a significantly high- 
Fig. 4. Time to diagnosis of mood disorder ( $n=950$ participants; $n=56$ events; rate $=$ 7.3 cases per 1,000 person-years).

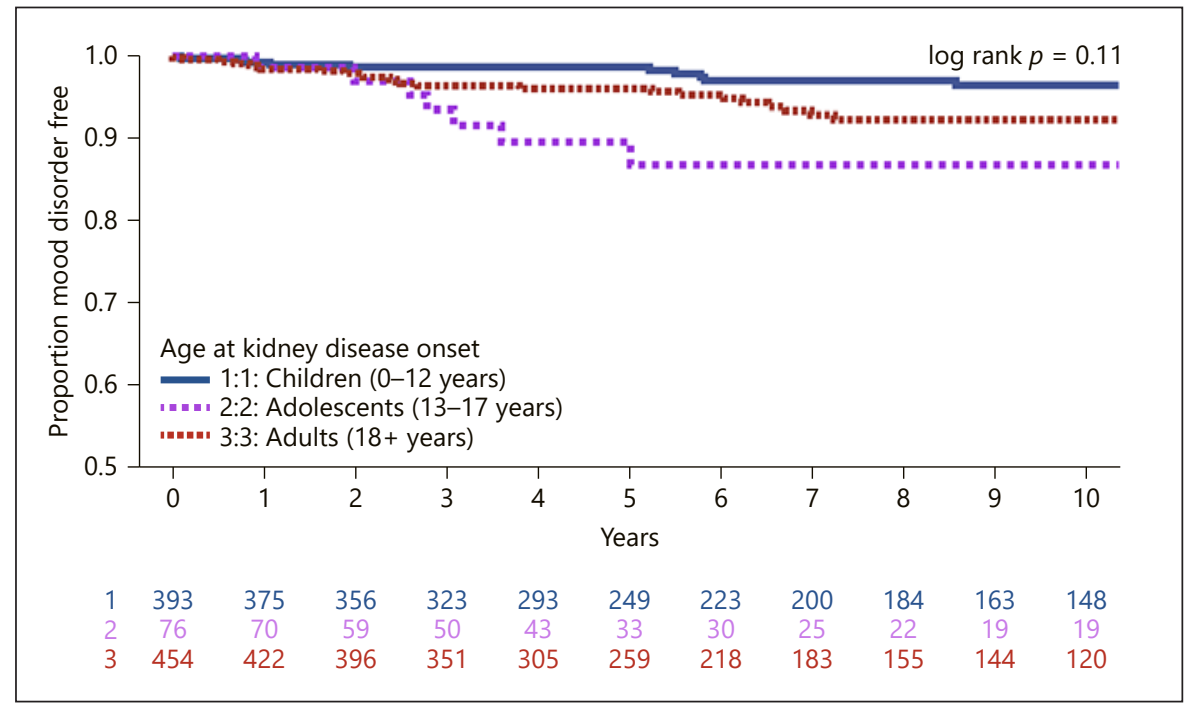

Table 2. Adjusted model results assessing the relationship between demographic and clinical characteristics and psychiatric diagnosis outcome

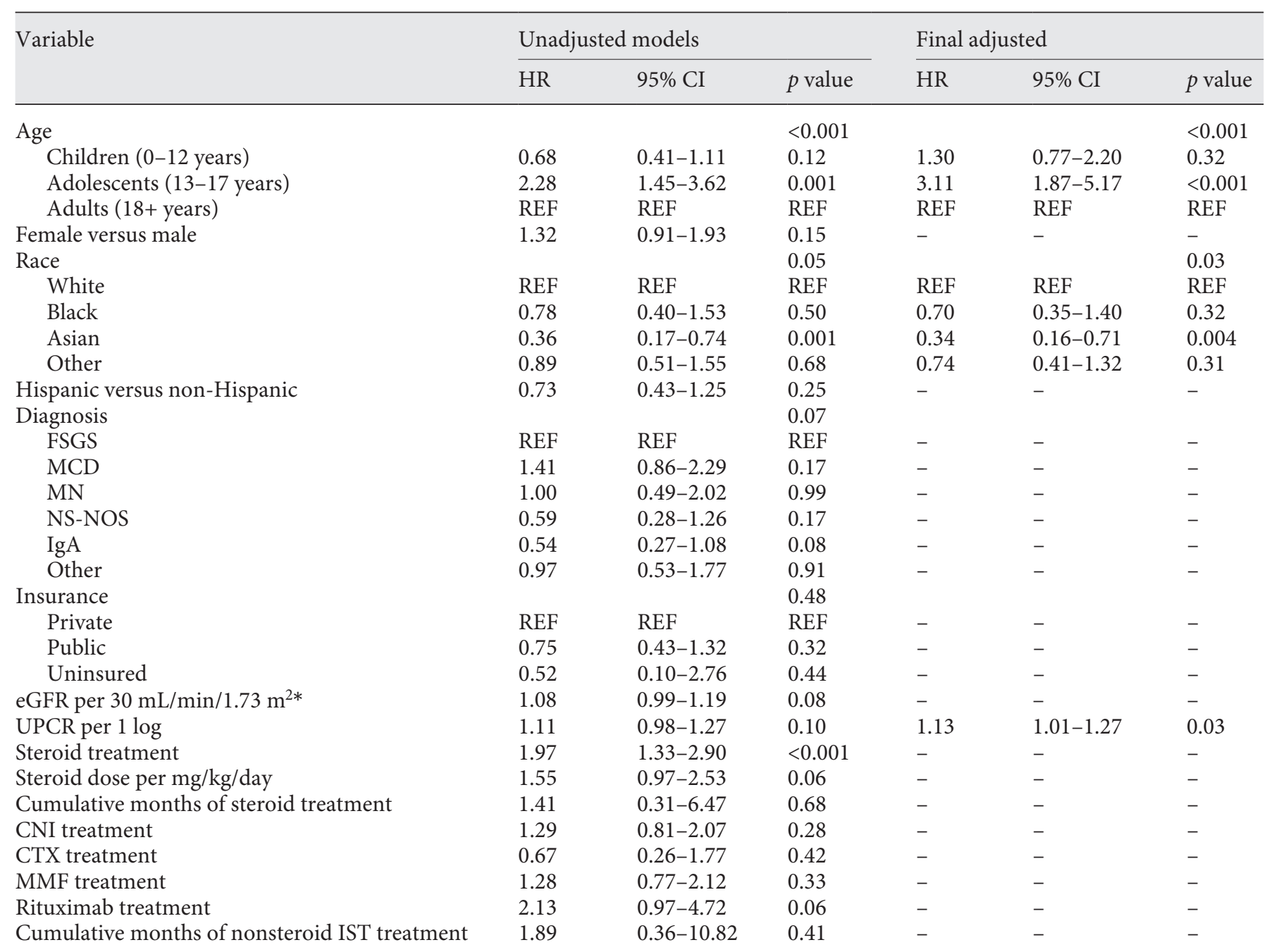


Table 2 (continued)

\begin{tabular}{|c|c|c|c|c|c|c|}
\hline Variable & \multicolumn{3}{|c|}{ Unadjusted models } & \multicolumn{3}{|c|}{ Final adjusted } \\
\hline Steroid response pattern & & & 0.84 & & & \\
\hline Sensitive & REF & REF & REF & - & - & - \\
\hline Dependent & 0.84 & $0.43-1.66$ & 0.62 & - & - & - \\
\hline Number of oral medications/day & & & $<0.001$ & & & $<0.001$ \\
\hline 0 & REF & REF & REF & REF & REF & REF \\
\hline 1 & 3.33 & $1.41-7.81$ & 0.006 & 3.36 & $1.41-8.02$ & 0.006 \\
\hline 2 & 2.67 & $1.01-7.11$ & 0.05 & 3.00 & $1.12-7.97$ & 0.03 \\
\hline $3-5$ & 7.33 & $4.11-13.06$ & $<0.001$ & 8.23 & $4.53-14.94$ & $<0.001$ \\
\hline
\end{tabular}

Analysis method: adjusted time-varying Cox proportional hazards model ( $n=943$ participants; $n=109$ events). FSGS, focal segmental glomerulosclerosis; MCD, minimal change disease; MN, membranous nephropathy; NS-NOS, nephrotic syndrome-not otherwise specified/unbiopsied; eGFR, estimated glomerular filtration rate; UPCR, urine protein-to-creatinine ratio; CNI, calcineurin inhibitors; CTX, Cytoxan; MMF, mycophenolate mofetil; IST, immunosuppressive therapy; HR, hazard ratio; CI, confidence interval. *HR reflects the hazard ratio of a $+30 \mathrm{~mL} / \mathrm{min} / 1.73 \mathrm{~m}^{2}$ difference in eGFR.

er rate of anxiety diagnosis compared to children and adults (children $=8.5$ vs. adolescents $=21.8$ vs. adults $=$ 9.3 cases per 1,000 person-years respectively, log-rank $p$ $<0.01)$. There was no difference in the rate of mood diagnosis by age (children $=5.3$ vs. adolescents $=12.4$ vs. adults $=8.5$ cases per 1,000 person-years, $\log$-rank $p=$ 0.11 ). Children and adolescents had higher rates of behavioral diagnosis, though this was based on a small $(n=$ 21 ) number of events (children $=4.2$ vs. adolescents $=3.5$ vs. adults $=1.1$ cases per 1,000 person-years respectively, $\log$-rank $p=0.03$ ).

Results of unadjusted Cox proportional hazard models are reported in Table 2. Adolescent age was associated with increased likelihood of psychiatric diagnosis compared with adults $(\mathrm{HR}=2.28,95 \% \mathrm{CI}=1.45-3.62)$. By race, Asians were less likely to be diagnosed with a psychiatric disorder compared with whites ( $\mathrm{HR}=0.36,95 \%$ $\mathrm{CI}=0.17-0.74)$. The onset of steroid treatment was also associated with diagnosis of psychiatric disorder (HR = $1.97,95 \%$ CI $=1.33-2.90$ ) but cumulative steroid dose and duration of steroid exposure were not (Table 2). Kaplan-Meier plots are shown with unadjusted analysis of anxiety, mood, and behavior outcomes by age in Figures 3 and 4 and online suppl. Figure 1, respectively.

Out of the 950 cases in this analysis, insurance status within 1 year of kidney diagnosis was only available in
486. While those with public insurance and the uninsured had lower onset of mood disorders, which was nonsignificant in this subanalysis, and insurance was not included in the multivariable model. There was no evidence of multicollinearity by age, eGFR, UPCR, and diagnosis, and all were included as independent variables in model selection (VIF: age $=1.50$, eGFR $=1.41, \mathrm{UPCR}=1.05$, $\mathrm{MCD}=1.01$, membranous nephropathy $=1.15$, nephrotic syndrome - not otherwise $=1.02, \operatorname{IgA}=1.10$, diagnosis - other $=1.00$, and FSGS $=$ reference) .

The final multivariable model was generated using backward selection for time to diagnosis of psychiatric disorder and results are shown in Table 2. Adolescents were more likely $(\mathrm{HR}=3.11,95 \% \mathrm{CI}=1.87-5.17)$ than adults to be diagnosed with psychiatric disorders. The differences by race persisted in this model, where Asians appeared to be at the lowest risk when white was used as the reference (HR $0.34,95 \% \mathrm{CI}=0.16-0.71$ ). Increasing UPCR was associated with an increase in risk for psychiatric disorder (HR 1.13, 95\% CI 1.01-1.27, per 1 log increase). The total number of oral medications per day was also associated with higher risk. In this multivariable model, steroid and other IST, kidney diagnosis, and eGFR were not associated with psychiatric disorder risk. 
Table 3. Adjusted model results assessing the relationship between psychiatric diagnosis and ESKD outcome

\begin{tabular}{llll}
\hline Adjusted model & $\begin{array}{l}\text { ESKD } \\
\text { HR }\end{array}$ & $95 \%$ CI & $p$ value \\
\hline $\begin{array}{l}\text { Onset of psychiatric disorder } \\
\text { Diagnosis }\end{array}$ & 2.45 & $1.53-3.92$ & $<0.001$ \\
$\quad$ FSGS & REF & REF & 0.008 \\
MCD & 0.65 & $0.41-1.05$ & REF \\
MN & 0.69 & $0.44-1.10$ & 0.12 \\
NS-NOS & 0.31 & $0.15-0.61$ & 0.001 \\
IgA & 0.71 & $0.53-0.96$ & 0.03 \\
Other & 0.77 & $0.54-1.11$ & 0.17 \\
eGFR at onset per $30 \mathrm{~mL} / \mathrm{min} / 1.73 \mathrm{~m}^{2 *}$ & 0.64 & $0.58-0.71$ & $<0.001$ \\
UPCR over time per $1 \log$ increase & 1.10 & $1.05-1.16$ & $<0.001$ \\
Steroid treatment & 0.61 & $0.47-0.81$ & $<0.001$ \\
\hline
\end{tabular}

Analysis method: adjusted time-varying Cox proportional hazards model $(n=943$ participants; $n=329$ events). ESKD, end-stage kidney disease; FSGS, focal segmental glomerulosclerosis; MCD, minimal change disease; MN, membranous nephropathy; NSNOS, nephrotic syndrome-not otherwise specified/unbiopsied; IgA, IgA nephropathy; eGFR, estimated glomerular filtration rate; UPCR, urine protein-to-creatinine ratio; HR, hazard ratio; $\mathrm{CI}$, confidence interval. ${ }^{*} \mathrm{HR}$ reflects the hazard ratio of a $+30 \mathrm{~mL} / \mathrm{min} / 1.73$ $\mathrm{m}^{2}$ difference in eGFR.

\section{End-Stage Kidney Disease}

Unadjusted and adjusted results of time to ESKD, including 330 ESKD events, are found in online suppl. Table 2 and Table 3, respectively. In univariate analysis, children were less likely to progress to ESKD (vs. adults, $\mathrm{HR}=0.46,95 \% \mathrm{CI}=0.36-0.59$ ), as were those with higher eGFR at onset (HR per $30 \mathrm{~mL} / \mathrm{min} / 1.73 \mathrm{~m}^{2}=0.59,95 \%$ $\mathrm{CI}=0.54-0.64)$. After adjustment for glomerular diagnosis, the eGFR at onset, UPCR over time, onset of steroid therapy, and onset of psychiatric disorder was associated with progression to ESKD ( $\mathrm{HR}=2.45,95 \% \mathrm{CI}=1.53-$ 3.92).

\section{Discussion/Conclusion}

This study was conducted to better understand the psychiatric disease burden, associated demographic and clinical variables, and relationship to ESKD in patients with glomerular disease. We found approximately 1 in 8 patients were diagnosed with a psychiatric disorder after the onset of kidney disease, and the rate was highest among adolescents (HR 3.11, 95\% CI 1.87-5.17). The HR of ESKD was greater following a psychiatric diagnosis, after adjusting for demographic and clinical characteristics (HR $=2.45,95 \%$ CI 1.53-3.9239).

To the best of our knowledge, this study represents the first investigation into the incidence psychiatric disorders among patients with nondiabetic glomerular diseases. The 12-month point prevalence of mood disorders is $9.5 \%$ in the USA for 18 years and older as reported by the National Comorbidity Survey Replication (NCS-R) [36]. Based on a previous systematic review and meta-analysis of interview-based depression diagnosis with a combined sample of roughly 56,000 participants [37], the prevalence of depression in advanced CKD stage 5 is approximately $22.8 \%$. Depression and CKD have been associated with poor outcomes including lower quality of life and death $[29,38]$. Previous population studies of patients with other chronic illnesses, including asthma and sickle cell disease, have found an increased prevalence of psychiatric disorders compared to the general population $[39,40]$.

In the current study, those of adolescent age at the time of kidney disease diagnosis showed the highest rate of psychiatric disorders: 33.4 cases per 1,000 person-years compared with other age-groups (children 13.3 and adults 13.2 cases per 1,000 person-years) as shown in Figure 2. The increased prevalence of psychiatric disorders among adolescents is seen in the general population as the proportion of adolescents age 13-18 years with mood disorders is $14.3 \%$, as reported by the NCS-R-Adolescent supplement [41]. According to the World Health Organization, adolescence is a critical time period for developing healthy social and emotional habits to support mental well-being. We posit that the additional stress of managing a chronic disease during adolescence places the popu- 
lation at increased risk of a psychiatric disorder. Population studies of adolescents with asthma have found increased likelihood of major depression (OR 1.7, 95\% CI 1.3-2.3) and anxiety disorders (OR 1.6, 95\% CI 1.2-2.2) compared to adolescents in the general community, which was postulated to be, in part, attributable to childhood adversity or unexamined familial factors [42]. These findings support the need for inclusion of mental health screening as part of chronic disease management, particularly among adolescents.

Steroid exposure was not associated with the onset of a psychiatric disorder. Rather in this analysis, higher UPCR and medication burden were associated with psychiatric disorder risk. The use of steroids has previously been shown to adversely affect mood as has been seen with an increase in manic symptoms in asthmatic patients treated with a short duration (7 days) of steroids [43]. In adults, the use of steroids has been shown to induce euphoria and hypomania in the short term. However, in the long term, the effects of steroid treatment tend to skew more toward depressive symptoms [44]. Furthermore, steroid dosage has been correlated with the incidence, but not severity, of psychiatric episodes in adults. The literature detailing child and adolescent psychiatric syndromes as a result of steroid use is not robust. The discordance of results in this study related to steroid use and psychiatric disease compared to prior publications may reflect the heretofore underappreciated impact of daily medication burden that more precisely reflect the medication - psychiatric disorder association. In addition, the use of diagnosed psychiatric disorders may have resulted in a lack of sensitivity to psychiatric disorders that are present but not diagnosed when associated with steroid therapy.

Systemic inflammation has been postulated to be a mechanism of psychiatric disease in some settings. Renal diseases such as lupus nephritis are systemic inflammatory diseases that can have psychiatric manifestations such as cognitive dysfunction, mood disorders, seizures, and cerebrovascular disease. Proposed mechanisms that cause these clinical manifestations include autoantibodies cross blood-brain barrier leading to neuronal injury [45]. Our sensitivity analysis revealed no difference in psychiatric disorder risk when lupus nephritis cases were removed from the analysis.

Our time-varying adjusted analyses of time to ESKD found that the onset of a psychiatric disorder was associated with ESKD. Previous literature has reported the presence of major depressive episodes in patients with progression to ESKD [29]. A study of 70 patients with ESKD demonstrated $71.4 \%$ had a DSM IV diagnosis of depression or anxiety [46]. Our study findings are consistent with the existing literature.

This study is not without limitations, namely, the reliance on EHR data from the KRN nephrology sites. Patients with a diagnosis of a psychiatric disorder that was not entered into the KRN-associated site EHR will not be recognized as cases in this study. Similarly, patients with an undiagnosed psychiatric disease would not be included as cases in this study. Our data collection relied on coded diagnoses and is likely an underrepresentation of the true prevalence of psychiatric disorders in our population. The ability to explore factors relevant to psychiatric diagnoses development, such as family history of psychiatric disorders and patient substance abuse disorders, is limited as family history was not collected in the study database and substance abuse may not be well-documented within EHRs. Finally, our aim was to investigate a possible association between glomerular disease and psychiatric disorders. We recognize that psychiatric disorders are complex and multifactorial in origin, including environmental, genetic, and socioeconomic factors.

Despite its limitations, this study suggests that diagnosed psychiatric illnesses are common in patients with glomerular disease. Future research and quality initiatives are recommended to systematically conduct mental health screening in patients across the age continuum with glomerular disease to define the true incidence and prevalence of psychiatric disorders in patients with glomerular disease and foster access clinical psychiatric support as indicated.

\section{Acknowledgement}

We are indebted to the patients and families who graciously participated in the Kidney Research Network (KRN) patient registry. We also thank our patient advisors for their encouragement on this topic and the study coordinators and data specialists at each of the participating KRN sites for their contributions that made this work possible. KRN sites: Cedars-Sinai Medical Center, Stanford University, Levine Children's Hospital, Los Angeles Biomedical Research Institute at Harbor-UCLA, Metrolina Nephrology Associates, and the Polyclinic, University of Michigan.

\section{Statement of Ethics}

This study was approved by the Institutional Review Board of the University of Michigan (reference number HUM00099659). Informed consent was obtained following institutional policies from participants or legal guardians of minor participants before enrollment in the study and consent covered future analyses of registry data. 


\section{Conflict of Interest Statement}

Though there are no conflicts of interest or disclosures related to these data, the authors would like to report the following financial relationships: J.P.T. has research funding through the University of Michigan (UM) with Retrophin Inc., Goldfinch Bio, Vertex Pharmaceuticals, and Pfizer Inc. D.S.G. has research funding through the UM with Retrophin Inc., Goldfinch Bio, Novartis, and Reata Pharmaceuticals, and consults through UM with Vertex and AstraZeneca. E.S.K. has research funding from Pfizer, has served as a one-time consultant for Mallinckrodt, and serves on the Board of NephCure Kidney International. S.F.M. serves on the Retrophin Advisory Board.

\section{Funding Sources}

The Kidney Research Network (KRN) registry enrollment, data collection, analytic design, interpretation of data, and writing of this manuscript are supported by the Atrium Health Medical Foundation and the University of Michigan. Additional analytic work for this study was funded in part by the National Center for Advancing Translational Sciences (NCATS Grant number: UL1TR002240) for the Michigan Institute for Clinical and Health Research.

\section{Author Contributions}

K.R.N. study design, conduct, and enrollment of participants: H.E.D., M.M., A.C., G.J.O., R.L., M.O., M.E., S.A., E.S.K., A.P., D.T.S., P.E.G., N.E.C., D.S.G., and S.F.M.; conception of overall research question: H.E.D.; statistical analyses: J.P.T.; and interpretation of results: all authors. All authors reviewed, revised, and approved the manuscript for submissions. Each author contributed important intellectual content during manuscript drafting and agreed to be personally accountable for the individual's own contributions and to ensure that questions pertaining to the accuracy or integrity of any portion of the work, even one in which the author was not directly involved, are appropriately investigated and resolved, including documentation in the literature if appropriate.

\section{References}

1 Fauman KR, Pituch KJ, Han YY, Niedner MF, Reske J, LeVine AM. Predictors of depressive symptoms in parents of chronically ill children admitted to the pediatric intensive care unit. Am J Hosp Palliat Care. 2011;28(8):55663.

2 Pinquart M, Shen Y. Depressive symptoms in children and adolescents with chronic physical illness: an updated meta-analysis. J Pediatr Psychol. 2011;36(4):375-84.

3 Schneider C, Jick SS, Bothner U, Meier CR COPD and the risk of depression. Chest. 2010;137(2):341-7.

4 Atlantis E, Fahey P, Cochrane B, Smith S. Bidirectional associations between clinically relevant depression or anxiety and COPD: a systematic review and meta-analysis. Chest. 2013;144(3):766-77.

5 Jolles MP, Haynes-Maslow L, Roberts MC, Dusetzina SB. Mental health service use for adult patients with co-occurring depression and physical chronic health care needs, 20072010. Med Care. 2015;53(8):708-12.

6 Hilliard ME, McQuaid EL, Nabors L, Hood $\mathrm{KK}$. Resilience in youth and families living with pediatric health and developmental conditions: introduction to the special issue on resilience. J Pediatr Psychol. 2015;40(9):8359.

7 Willgoss TG, Yohannes AM. Anxiety disorders in patients with COPD: a systematic review. Respir Care. 2013;58(5):858-66.

8 Balhara YP. Diabetes and psychiatric disorders. Indian J Endocrinol Metab. 2011;15(4): 274-83.

9 Jonassaint CR, Jones VL, Leong S, Frierson GM. A systematic review of the association between depression and health care utiliza- tion in children and adults with sickle cell disease. Br J Haematol. 2016;174(1):136-47.

10 Sogutlu A, Levenson JL, McClish DK, Rosef $\mathrm{SD}$, Smith WR. Somatic symptom burden in adults with sickle cell disease predicts pain, depression, anxiety, health care utilization, and quality of life: the PiSCES project. Psychosomatics. 2011;52(3):272-9.

11 Goodwin RD, Robinson M, Sly PD, McKeague IW, Susser ES, Zubrick SR, et al. Severity and persistence of asthma and mental health: a birth cohort study. Psychol Med. 2013;43(6):1313-22.

12 Hijmans CT, Grootenhuis MA, Oosterlaan J, Last BF, Heijboer H, Peters M, et al. Behavioral and emotional problems in children with sickle cell disease and healthy siblings: multiple informants, multiple measures. Pediatr Blood Cancer. 2009;53(7):1277-83.

13 Barlow JH, Ellard DR. The psychosocial wellbeing of children with chronic disease, their parents and siblings: an overview of the research evidence base. Child Care Health Dev. 2006;32(1):19-31.

14 Scott KM, Von Korff M, Ormel J, Zhang MY, Bruffaerts R, Alonso J, et al. Mental disorders among adults with asthma: results from the World Mental Health Survey. Gen Hosp Psychiatry. 2007;29(2):123-33.

15 Opolski M, Wilson I. Asthma and depression: a pragmatic review of the literature and recommendations for future research. Clin Pract Epidemiol Ment Health. 2005; 1:18.

16 Kankaanranta H, Kauppi P, Tuomisto LE, Ilmarinen P. Emerging comorbidities in adult asthma: risks, clinical associations, and mechanisms. Mediators Inflamm. 2016;2016: 3690628.
17 Knudsen KB, Pressler T, Mortensen LH, Jarden M, Skov M, Quittner AL, et al. Associations between adherence, depressive symptoms and health-related quality of life in young adults with cystic fibrosis. Springerplus. 2016;5(1):1216.

18 Yohannes AM, Willgoss TG, Fatoye FA, Dip MD, Webb K. Relationship between anxiety, depression, and quality of life in adult patients with cystic fibrosis. Respir Care. 2012;57(4): 550-6.

19 Riekert KA, Bartlett SJ, Boyle MP, Krishnan JA, Rand CS. The association between depression, lung function, and health-related quality of life among adults with cystic fibrosis. Chest. 2007;132(1):231-7.

20 Substance Abuse and Mental Health Services Administration. 2018 National Survey on Drug Use and Health: methodological summary and definitions. Rockville, MD: Center for Behavioral Health Statistics and Quality, Substance Abuse, and Mental Health Services Administration; 2019.

21 Ghandour RM, Sherman LJ, Vladutiu CJ, Ali MM, Lynch SE, Bitsko RH, et al. Prevalence and treatment of depression, anxiety, and conduct problems in US children. J Pediatr. 2019;206:256-e3.

22 Malik JA, Koot HM. Explaining the adjustment of adolescents with type 1 diabetes: role of diabetes-specific and psychosocial factors. Diabetes Care. 2009;32(5):774-9.

23 Lin EH, Von Korff M, Alonso J, Angermeyer MC, Anthony J, Bromet E, et al. Mental disorders among persons with diabetes: results from the World Mental Health Surveys. J Psychosom Res. 2008;65(6):57180. 
24 Ducat L, Philipson LH, Anderson BJ. The mental health comorbidities of diabetes. JAMA. 2014;312(7):691-2.

25 Anderson RJ, Freedland KE, Clouse RE, Lustman PJ. The prevalence of comorbid depression in adults with diabetes: a meta-analysis. Diabetes Care. 2001;24(6):1069-78.

26 Nouwen A, Lloyd CE, Pouwer F. Depression and type 2 diabetes over the lifespan: a metaanalysis. Response to Mezuk et al. Diabetes Care. 2009;32(5):e56; author reply e7. .

27 Goh ZS, Griva K. Anxiety and depression in patients with end-stage renal disease: impact and management challenges: a narrative review. Int J Nephrol Renovasc Dis. 2018;11: 93-102.

28 Kimmel PL, Peterson RA. Depression in patients with end-stage renal disease treated with dialysis: has the time to treat arrived? Clin J Am Soc Nephrol. 2006;1(3):349-52.

29 Hedayati SS, Minhajuddin AT, Afshar M Toto RD, Trivedi MH, Rush AJ. Association between major depressive episodes in patients with chronic kidney disease and initiation of dialysis, hospitalization, or death. JAMA. 2010;303(19):1946-53.

30 Hedayati SS, Minhajuddin AT, Toto RD, Morris DW, Rush AJ. Prevalence of major depressive episode in CKD. Am J Kidney Dis. 2009;54(3):424-32.

31 Cukor D, Fruchter Y, Ver Halen N, Naidoo S, Patel A, Saggi SJ. A preliminary investigation of depression and kidney functioning in patients with chronic kidney disease. Nephron Clin Pract. 2012;122(3-4):139-45.
32 Kogon AJ, Matheson MB, Flynn JT, Gerson AC, Warady BA, Furth SL, et al. Depressive symptoms in children with chronic kidney disease. J Pediatr. 2016;168:164-e1.

33 Gipson DS, Selewski DT, Massengill SF, Modes MM, Desmond H, Lee L, et al. NephCure accelerating cures institute: a multidisciplinary consortium to improve care for $\mathrm{Ne}$ phrotic syndrome. Kidney Int Rep. 2018;3(2): $439-46$.

34 Schwartz GJ, Muñoz A, Schneider MF, Mak RH, Kaskel F, Warady BA, et al. New equations to estimate GFR in children with CKD. J Am Soc Nephrol. 2009;20(3):629-37.

35 Levey AS, Stevens LA, Schmid CH, Zhang YL, Castro AF 3rd, Feldman HI, et al. A new equation to estimate glomerular filtration rate. Ann Intern Med. 2009;150(9):604-12.

36 Kessler RC, Chiu WT, Demler O, Merikangas $\mathrm{KR}$, Walters EE. Prevalence, severity, and comorbidity of 12-month DSM-IV disorders in the National Comorbidity Survey Replication. Arch Gen Psychiatry. 2005;62(6):61727.

37 Palmer S, Vecchio M, Craig JC, Tonelli M, Johnson DW, Nicolucci A, et al. Prevalence of depression in chronic kidney disease: systematic review and meta-analysis of observational studies. Kidney Int. 2013;84(1):179-91.

38 Bautovich A, Katz I, Smith M, Loo CK, Harvey SB. Depression and chronic kidney disease: a review for clinicians. Aust N Z J Psychiatry. 2014;48(6):530-41.
39 Ortega AN, Huertas SE, Canino G, Ramirez R, Rubio-Stipec M. Childhood asthma, chronic illness, and psychiatric disorders. J Nerv Ment Dis. 2002;190(5):275-81.

40 Unal S, Toros F, Kütük MÖ, Uyanıker MG. Evaluation of the psychological problems in children with sickle cell anemia and their families. Pediatr Hematol Oncol. 2011;28(4):3218.

41 Merikangas KR, He JP, Burstein M, Swanson SA, Avenevoli S, Cui L, et al. Lifetime prevalence of mental disorders in U.S. adolescents: results from the National Comorbidity Survey Replication-Adolescent Supplement (NCS-A). J Am Acad Child Adolesc Psychiatry. 2010;49(10):980-9.

42 Goodwin RD, Fergusson DM, Horwood LJ. Asthma and depressive and anxiety disorders among young persons in the community. Psychol Med. 2004;34(8):1465-74.

43 Kewalramani A, Bollinger ME, Postolache TT. Asthma and mood disorders. Int J Child Health Hum Dev. 2008;1(2):115-23.

44 Warrington TP, Bostwick JM. Psychiatric adverse effects of corticosteroids. Mayo Clin Proc. 2006;81(10):1361-7.

45 Kivity S, Agmon-Levin N, Zandman-Goddard G, Chapman J, Shoenfeld Y. Neuropsychiatric lupus: a mosaic of clinical presentations. BMC Med. 2015;13:43.

46 Cukor D, Coplan J, Brown C, Friedman S, Cromwell-Smith A, Peterson RA, et al. Depression and anxiety in Urban hemodialysis patients. Clin J Am Soc Nephrol. 2007;2(3): 484-90. 